\title{
A importância do papel do enfermeiro na humanização do parto: verificação completiva
}

The importance of the nurse's role in the humanization of childbirth: comprehensive verification

La importancia del papel de la enfermera en la humanización del parto: verificación integral

Aline Barreto Hora

ORCID: https://orcid.org/0000-0002-3930-6475 Centro Universitário Estácio de Sergipe, Brasil

E-mail: aline.barretoh@ hotmail.com

Anne Karolyne Silva Santos

ORCID: https://orcid.org/0000-0003-0798-6207 Centro Universitário Estácio de Sergipe, Brasil

E-mail: anne_karolyne03@hotmail.com

Cristiane Santana de Oliveira

ORCID: https://orcid.org/0000-0001-5811-9758

Centro Universitário Estácio de Sergipe, Brasil

E-mail: tianeoliveira2010@hotmail.com

Maiza Conceição Gonzaga de Oliveira Feitosa

ORCID: https://orcid.org/0000-0001-7882-5713

Centro Universitário Estácio de Sergipe, Brasil

E-mail: maiza_gonzaga@ hotmail.com

Weber de Santana Teles

ORCID: https://orcid.org/0000-0003-1770-8278

Centro de Hemoterapia de Sergipe, Brasil

E-mail: arteecura@ hotmail.com

Max Cruz da Silva

ORCID: https://orcid.org/0000-0002-6944-5986 Faculdade Pio Décimo, Brasil

E-mail: maxlfi@ hotmail.com

Taíssa Alice Soledade Calasans

ORCID: https://orcid.org/0000-0003-0460-4437 Universidade Tiradentes, Brasil

Ângela Maria Melo Sá Barros

ORCID: https://orcid.org/0000-0003-4087-3247

Universidade Federal do Rio de Janeiro, Brasil E-mail: angelsamelo@hotmail.com

Ruth Cristini Torres

ORCID: https://orcid.org/0000-0002-8664-192X Instituto de Hematologia e Hemoterapia de Sergipe, Brasil

E-mail: ruthcristini@gmail.com

Marcel Vinícius Cunha Azevedo ORCID: https://orcid.org/0000-0002-5312-3333

Centro Universitário Estácio Sergipe, Brasil

E-mail: marcelvinicius49@gmail.com

Ana Fátima Souza Melo de Andrade

ORCID: https://orcid.org/0000-0002-7024-6175

Centro Universitário Estácio de Sergipe, Brasil

E-mail: anafatimamelo@hotmail.com

Paulo Celso Curvelo Santos Junior ORCID: https://orcid.org/0000-0001-5834-6782

Universidade Tiradentes, Brasil

E-mail: paulo.curvelo.jr@gmail.com

Alejandra Debbo

ORCID: https://orcid.org/0000-0002-7743-5921

Universidade Tiradentes, Brasil

E-mail: aledebbo@hotmail.com

Maria Hozana Santos Silva

ORCID: https://orcid.org/0000-0001-5742-5366

Faculdade Ages de Medicina, Brasil

E-mail: hosana_p@hotmail.com 


\begin{abstract}
Resumo
Trata-se de uma revisão integrativa que foram iniciadas as buscas através das escolhas dos artigos científicos publicados entre os anos de 2016 a 2021. Portanto, através dos resultados trazidos nesse estudo, tem-se a identificação e descrição com a síntese dos artigos que tratam sobre o assunto abordado na presente pesquisa. De acordo com Lima et al. (2020), a participação efetiva da enfermeira obstétrica no acompanhamento do trabalho de parto e nascimento qualifica o trabalho em equipe e contribui para um cuidado humanizado. O desenvolvimento do trabalho permitiu compreender que o enfermeiro obstetra possui conhecimentos técnicos necessários para o desenvolvimento dessa prática, principalmente por ser situação em que o médico não se encontra totalmente presente ou por não poder estar durante todo o processo em função do tempo que se leva. Com isso, o profissional poderá utilizar da sua preparação profissional para atuar diretamente com a gestante, prestando todos os cuidados e esclarecimentos necessários, conversando, tranquilizando, indicando as opções e estimulando a parturiente para que ela enfrente o parto com conforto, segurança e menos dor.
\end{abstract}

Palavras-chave: Parto humanizado; Papel do profissional de enfermagem; Humanização da assistência.

\begin{abstract}
It is an integrative review that started the searches through the choices of scientific articles published between the years 2016 to 2021 . Therefore, through the results brought in this study, there is the identification and description with the synthesis of the articles that deal with on the subject addressed in this research. According to Lima et al. (2020), the effective participation of the obstetric nurse in monitoring labor and birth qualifies teamwork and contributes to humanized care. The development of the work allowed us to understand that the obstetric nurse has the necessary technical knowledge for the development of this practice, mainly because it is a situation in which the doctor is not fully present or because he cannot be during the entire process due to the time it takes. With this, the professional will be able to use their professional preparation to work directly with the pregnant woman, providing all the necessary care and clarification, talking, reassuring, indicating the options and encouraging the parturient to face the birth with comfort, safety and less pain.
\end{abstract}

Keywords: Humanized delivery; Role of the nursing professional; Humanization of assistance.

\title{
Resumen
}

Se trata de una revisión integradora que inició las búsquedas a través de la elección de artículos científicos publicados entre los años 2016 al 2021. Por tanto, a través de los resultados aportados en este estudio, se encuentra la identificación y descripción con la síntesis de los artículos que tratan sobre el tema abordado en esta investigación. Según Lima et al. (2020), la participación efectiva de la enfermera obstétrica en el seguimiento del trabajo de parto y el nacimiento capacita el trabajo en equipo y contribuye a la atención humanizada. El desarrollo del trabajo permitió entender que la enfermera obstétrica cuenta con los conocimientos técnicos necesarios para el desarrollo de esta práctica, principalmente por tratarse de una situación en la que el médico no está plenamente presente o porque no puede estar durante todo el proceso debido al tiempo que lleva. Con ello, el profesional podrá utilizar su preparación profesional para trabajar directamente con la gestante, brindándole todos los cuidados y aclaraciones necesarias, hablando, tranquilizando, indicando las opciones y animando a la parturienta a afrontar el parto con comodidad, seguridad y menos dolor.

Palabras clave: Parto humanizado; Rol del profesional de enfermería; Humanización de la asistencia.

\section{Introdução}

As transformações sociais, econômicas e políticas influem diretamente na vida da mulher durante o puerpério, pois a gravidez representa uma etapa única na vida da mesma, onde a mesma passa tanto por transformações físicas como psicológicas, gerando grandes expectativas e dúvidas que influenciarão de forma direta na saúde e no bem-estar (Barbosa et al., 2018 \& Cabral et al., 2020).

Portanto, o parto é considerado um processo natural e fisiológico, que esteve presente na vida da mulher desde sempre (Mendonça et al., 2021). Sendo assim, com as mudanças voltadas à esta prática, o parto humanizado, é considerado uma prática relacionada ao cuidado a mãe e o bebê durante o pré-natal até a sua chegada, garantindo uma qualidade de assistência segura tencionando a valorização e a escolha do ato de dar à luz de forma natural e que a parentela faça parte dessa experiência (Pereira et al., 2016 \& Silva et al., 2021).

As práticas humanizadas consistem em oferecer à gestante e a família, apoio e informações necessárias para o trabalho de parto e parição qualificada. Incluem cuidados como: orientar a gestante quanto à alimentação, deambulação, contrações dolorosas e escolha da posição pela paciente durante a concepção e o parto, direito ao acompanhante e local do nascimento (Ferreira et al., 2019 \& Cananéa et al., 2020). 
O Ministério da Saúde (MS) criou, em 2011, a Rede Cegonha e em 2017, lançou as Diretrizes Nacionais de Assistência ao Parto Normal, que nasceram de um esforço do Ministério da Saúde, por meio da Coordenação-Geral de Saúde da Mulher do Departamento de Ações Programáticas Estratégicas, da Secretaria de Atenção à Saúde do Ministério da Saúde (CGSM/DAPES/ SAS/MS), em conjunto com diversas áreas (Mendes, 2019).

Diante o exposto, ambos projetos têm como intuito prioritário de garantir uma assistência humanizada e qualificada na gravidez, parto e puerpério, assim como promover uma assistência de qualidade ao recém-nascido, difundindo as práticas baseadas em evidências, alinhando a assistência prestada, trazendo mudanças nas práticas clínicas e reduzindo as intervenções desnecessárias. Ressalta-se, no entanto a importância do papel do enfermeiro e outros profissionais da saúde da área obstetra por serem responsáveis pela redução de intervenções e por promoverem a maior satisfação entre as mulheres atendidas.

Entretanto, entendemos que a humanização e orientação deve ser iniciada na primeira consulta de pré-natal, pois assim, as gestantes podem se preparar física, psicológica e emocionalmente para que a assistência ao trabalho de parto seja menos estressante (Nascimento et al., 2020).

O estudo torna-se relevante como proposta para refletir sobre o conceito do parto humanizado, apontando as contribuições do nascimento humanizado às parturientes e objetiva-se analisar através da literatura o papel do enfermeiro durante a assistência ao parto humanizado.

\section{Metodologia}

Trata-se de uma revisão integrativa de caráter exploratório e descritivo com enfoque qualitativo, a qual foi realizada no período de fevereiro a maio/2021. A revisão integrativa da literatura, que constitui uma ferramenta importante permitindo a análise de subsídios na literatura de forma ampla, além de divulgar dados científicos produzidos por outros autores. Destaca-se pela exigência dos mesmos padrões de rigor, clareza e replicação utilizados em estudos primários, além de compor a mais ampla abordagem metodológica referente às revisões de literatura (Cerqueira et al., 2018).

Para execução do estudo foram realizadas as seguintes etapas: 1) delimitação do tema, 2) seleção dos descritores, 3) estabelecimento dos critérios de inclusão e exclusão, 4) elaboração dos objetivos geral e específicos, e 5) seleção de artigos. Utilizaram-se como critério de inclusão, artigos originais, na linguagem portuguesa, respeitando os limites de publicação entre 2016 a 2021, com acesso livre e disponível em texto completo online.

A coleta dos dados foi iniciada em junho a dezembro de 2020, sendo realizada por meio das bases de dados bases de dados Scientific Electronic Library Online (SciELO), Biblioteca Virtual da Saúde (BVS) e Google Acadêmico, e abrangeram os descritores da saúde (Decs): "Parto humanizado"; "Papel do profissional de enfermagem"; "Humanização da assistência".

Foram utilizados artigos publicados nos anos de 2016 a 2021 em concordância com os critérios que incluem estudos em português, inglês e espanhol, que tratam sobre o respectivo tema referente à humanização do parto, ainda os que possuem dados e informações esperadas, mediante os objetivos, critérios de inclusão e exclusão, na qual serão incluídos os artigos com textos completos, originais e pertinentes ao estudo. Desse modo, servirão como fatores de exclusão, os seguintes aspectos: aqueles que não fazem alusão ao tema e que não estão disponibilizados na versão completa, para então da continuidade com a leitura na íntegra.

Após a delimitação dos estudos foram realizadas a leitura, sistematização e construção do material preservando os critérios éticos, no qual, todos os autores consultados serão referenciados ao longo da pesquisa, conservando a fidedignidade das informações utilizadas. Na Figura 1 está apresentado o fluxograma na modalidade prisma que foi utilizado para construção da revisão integrativa. 
Figura 1 - Fluxograma na modalidade prisma das etapas metodológicas empregadas na revisão integrativa.

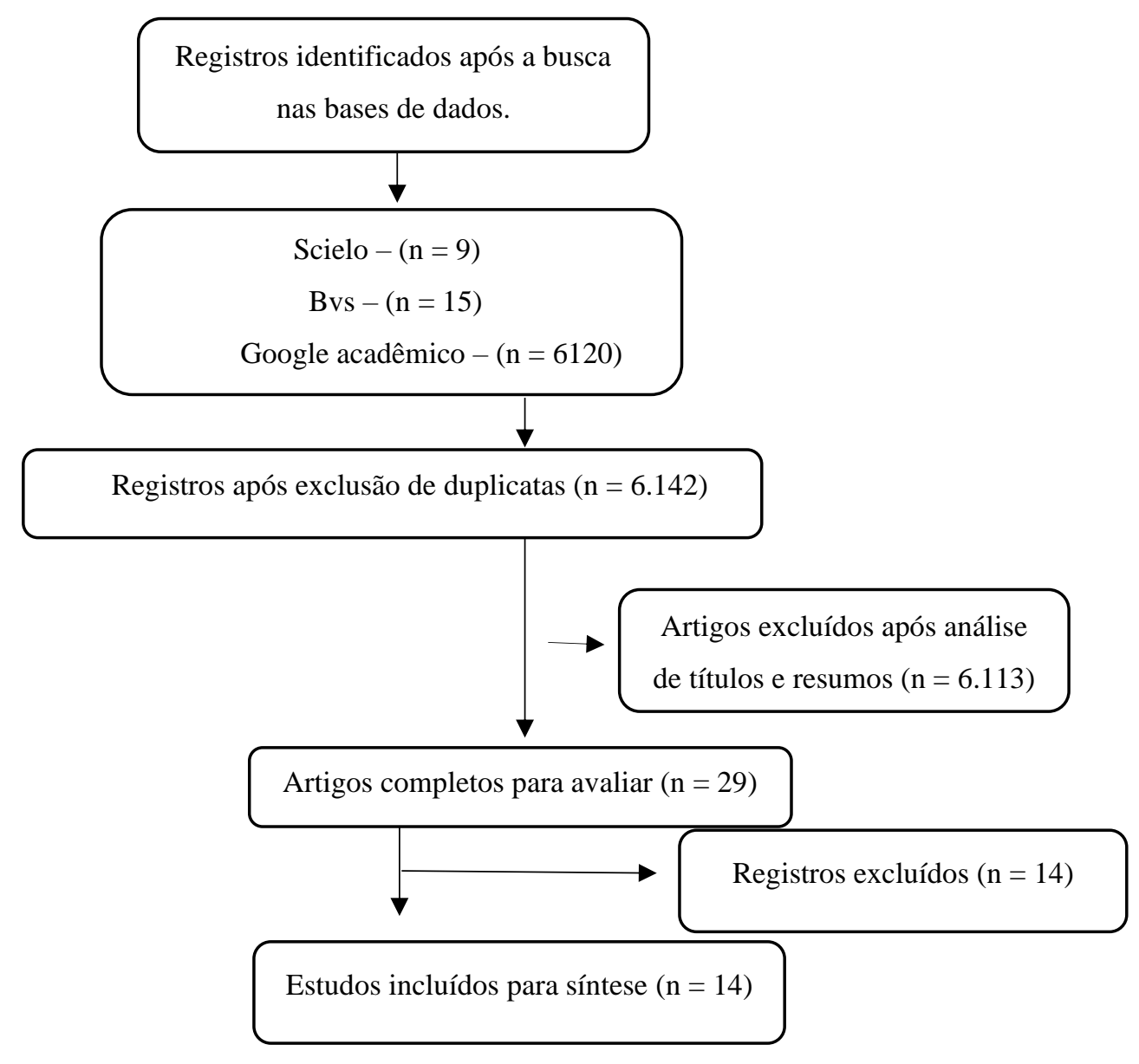

Fonte: Autores (2021).

\section{Resultados e Discussão}

Os artigos trazem definições sobre o processo de parturição, assistência da enfermagem no processo do parto humanizado, atuação do enfermeiro, percepção das puérperas sobre o parto e cuidados de enfermagem nesse processo. Portanto, através dos resultados trazidos nesse estudo, tem-se a identificação e descrição com a síntese dos artigos que tratam sobre o assunto abordado na presente pesquisa (Quadro 1).

Quadro 1 - Síntese dos artigos selecionados para a discussão dos resultados acerca do assunto.

\begin{tabular}{|c|c|c|c|c|}
\hline $\mathbf{N}^{0}$ & $\begin{array}{l}\text { AUTOR/ } \\
\text { ANO }\end{array}$ & TÍTULO DO ARTIGO & TIPO DE ESTUDO & SÍNTESE DO ESTUDO \\
\hline 1 & $\begin{array}{l}\text { Pereira, S.S., } \\
\text { Oliveira, I.C.M.S., Santos, } \\
\text { J.B.S.S., \& } \quad \text { Carvalho, } \\
\text { M.C.M.P. 2016. }\end{array}$ & $\begin{array}{l}\text { Parto natural: a atuação do } \\
\text { enfermeiro diante da assistência } \\
\text { humanizada. }\end{array}$ & $\begin{array}{l}\text { Uma pesquisa descritiva } \\
\text { com abordagem } \\
\text { qualitativa. }\end{array}$ & $\begin{array}{l}\text { O parto é um processo natural e } \\
\text { fisiológico que desde os primórdios } \\
\text { era realizado de maneira } \\
\text { instintiva pela mulher e auxiliado por } \\
\text { pessoas que tinham certo } \\
\text { conhecimento sobre o nascimento. }\end{array}$ \\
\hline 2 & Possati, A.B et al. 2017. & $\begin{array}{l}\text { Humanização do parto: } \\
\text { significados e percepções de } \\
\text { Enfermeiras. }\end{array}$ & $\begin{array}{l}\text { Pesquisa qualitativa } \\
\text { descritiva. }\end{array}$ & $\begin{array}{l}\text { Traz a história do parto e das } \\
\text { modificações sofridas com o passar } \\
\text { dos anos. }\end{array}$ \\
\hline 3 & Lima, P.C et al. 2017. & 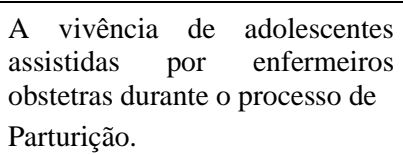 & $\begin{array}{l}\text { Pesquisa descritiva, } \\
\text { exploratória, de natureza } \\
\text { qualitativa. }\end{array}$ & $\begin{array}{l}\text { Traz a respeito do parto na } \\
\text { adolescência, o relato de parto das } \\
\text { adolescentes. }\end{array}$ \\
\hline
\end{tabular}




\begin{tabular}{|c|c|c|c|c|}
\hline 4 & Barros, F.R.B et al. 2018. & $\begin{array}{l}\text { Percepção das puérperas } \\
\text { manauaras frente À assistência } \\
\text { de enfermagem no preparo do } \\
\text { Trabalho de parto e nascimento }\end{array}$ & $\begin{array}{l}\text { Estudo do tipo descritivo e } \\
\text { exploratório } \quad \text { de } \\
\text { abordagem mista. }\end{array}$ & $\begin{array}{l}\text { Destaca a importância do enfermeiro } \\
\text { no acompanhamento a parturiente. }\end{array}$ \\
\hline 5 & Martins, D.O et al. 2018. & $\begin{array}{l}\text { Percepção das puérperas sobre o } \\
\text { parto normal humanizado }\end{array}$ & $\begin{array}{lr}\text { Estudo do tipo descritivo, } \\
\text { exploratório } \\
\text { abordagem } & \text { com } \\
\text { qualitativa. } & \text { quanti- }\end{array}$ & $\begin{array}{l}\text { Traz sobre a descoberta da gravidez e } \\
\text { o procedimento realizado, diz como o } \\
\text { acolhimento é importante para esse } \\
\text { momento. }\end{array}$ \\
\hline 6 & $\begin{array}{l}\text { Alves, T.T.M et al. } \\
2018 .\end{array}$ & $\begin{array}{l}\text { Atuação da enfermeira obstetra } \\
\text { no desenrolar do trabalho de } \\
\text { parto e parto. }\end{array}$ & $\begin{array}{l}\text { Trata-se de uma pesquisa } \\
\text { descritiva, exploratório de } \\
\text { abordagem qualitativa. }\end{array}$ & $\begin{array}{l}\text { A enfermeira obstetra deve seguir um } \\
\text { modelo de assistência que busque } \\
\text { resgatar valores como o } \\
\text { protagonismo, a individualidade, a } \\
\text { privacidade e a autonomia da mulher, } \\
\text { objetivando a promoção de partos } \\
\text { saudáveis, eliminando intervenções } \\
\text { desnecessárias e oferecendo outras } \\
\text { comprovadamente benéficas. }\end{array}$ \\
\hline 7 & $\begin{array}{l}\text { Mota, B.R., Waltrick, } \\
\text { M.A.M., \& Barbosa, T.M. } \\
\text { 2019. }\end{array}$ & $\begin{array}{l}\text { Mulheres em puerpério: } \\
\text { representação social sobre o } \\
\text { atendimento da enfermagem no } \\
\text { momento do parto }\end{array}$ & $\begin{array}{l}\text { Pesquisa com abordagem } \\
\text { qualitativa. }\end{array}$ & $\begin{array}{l}\mathrm{O} \text { ato fisiológico do parir com o } \\
\text { passar dos anos deixou de ser visto } \\
\text { como algo patológico e deu privilegio } \\
\text { a técnica mecanizada. }\end{array}$ \\
\hline 8 & $\begin{array}{l}\text { Ahlert, E.C., Alves, A.M., \& } \\
\text { Lohmann, P.M. } 2019 .\end{array}$ & $\begin{array}{l}\text { A percepção dos profissionais da } \\
\text { área Obstétrica de dois hospitais } \\
\text { do vale do taquari Sobre o parto } \\
\text { humanizado }\end{array}$ & $\begin{array}{l}\text { Trata-se de uma pesquisa } \\
\text { de caráter exploratório- } \\
\text { descritivo com abordagem } \\
\text { qualitativa e quantitativa. }\end{array}$ & $\begin{array}{l}\text { As modificações do parto ao longo } \\
\text { dos anos em relação ao modelo } \\
\text { assistencial. }\end{array}$ \\
\hline 9 & $\begin{array}{l}\text { Santana, A.T., } \\
\text { Felzemburgh, R.D.M., } \\
\text { Couto, T.M., } \\
\text { Pereira, L.P. } \\
2019\end{array}$ & $\begin{array}{l}\text { Atuação de enfermeiras } \\
\text { residentes em obstetrícia na } \\
\text { assistência ao parto. }\end{array}$ & $\begin{array}{l}\text { Estudo transversal } \\
\text { descritivo com abordagem } \\
\text { quantitativa. }\end{array}$ & $\begin{array}{l}\text { O Programa de Residência em } \\
\text { Enfermagem, ponto importante no } \\
\text { processo de humanização do parto, } \\
\text { associa-se diretamente ao aumento } \\
\text { dos índices de partos normais, maior } \\
\text { utilização de boas práticas na } \\
\text { assistência ao parto e redução das } \\
\text { intervenções obstétricas. }\end{array}$ \\
\hline 10 & Braz, I.M.A et al. 2019. & $\begin{array}{l}\text { Interdisciplinaridade na } \\
\text { assistência ao parto: percepção } \\
\text { dos enfermeiros obstetras. }\end{array}$ & $\begin{array}{l}\text { Trata-se de um estudo } \\
\text { qualitativo, } \\
\text { exploratório. }\end{array}$ & $\begin{array}{l}\text { Diz sobre a mortalidade materna e } \\
\text { retrata a grande dificuldade a } \\
\text { assistência de saúde no Brasil. }\end{array}$ \\
\hline 11 & Piler, A.A et al. 2020. & $\begin{array}{l}\text { Cuidados no processo de } \\
\text { parturição sob a ótica dos } \\
\text { profissionais de enfermagem }\end{array}$ & $\begin{array}{l}\text { Estudo com abordagem } \\
\text { qualitativa. }\end{array}$ & $\begin{array}{l}\text { Aborda as políticas públicas voltadas } \\
\text { à atuação da enfermagem obstétrica } \\
\text { na assistência ao parto e nascimento e } \\
\text { da necessidade da mudança para o } \\
\text { modelo na atenção obstétrica } \\
\text { centrado na mulher. }\end{array}$ \\
\hline 12 & Santos, J.C.M et al. 2020. & $\begin{array}{l}\text { A percepção das puérperas sobre } \\
\text { o parto vaginal humanizado } \\
\text { assistido pela equipe de } \\
\text { Enfermagem }\end{array}$ & $\begin{array}{l}\text { Trata-se de um estudo } \\
\text { descritivo, } \\
\text { abordagem qualitativa. }\end{array}$ & $\begin{array}{l}\text { Traz sobre a prática da humanização, } \\
\text { o papel e a importância do enfermeiro } \\
\text { obstetra. }\end{array}$ \\
\hline 13 & Silva, M.J et al. 2020. & $\begin{array}{l}\text { O movimento pela humanização } \\
\text { do parto e nascimento no Brasil: } \\
\text { o impacto em Uberlândia } \\
\text { segundo a percepção dos } \\
\text { enfermeiros }\end{array}$ & $\begin{array}{l}\text { Estudo observacional, } \\
\text { descritivo, de abordagem } \\
\text { quali-quantitativa }\end{array}$ & $\begin{array}{l}\text { Medicalização no Brasil para o parto, } \\
\text { dando prioridade a cesáreas. }\end{array}$ \\
\hline 14 & $\begin{array}{l}\text { Lima, M.M., } \\
\text { Ribeiro, L.N., } \\
\text { Costa, R., } \\
\text { Monguilhot, } \\
\text { J.J.C., } \\
\text { Gomes, I.E.M. } \\
2020 .\end{array}$ & $\begin{array}{l}\text { Enfermeiras obstétricas no } \\
\text { processo de parturição: } \\
\text { percepção das mulheres. }\end{array}$ & $\begin{array}{l}\text { Estudo qualitativo, } \\
\text { descritivo-exploratório. }\end{array}$ & $\begin{array}{llr}\text { Define o parto e como ele é } \\
\text { apresentado no Brasil, } & \text { as } \\
\text { experiencias vivenciadas } & \text { por } \\
\text { mulheres e a participação } & \text { da } \\
\text { enfermeira obstétrica } & \text { no } \\
\text { acompanhamento do trabalho de } \\
\text { parto. }\end{array}$ \\
\hline 15 & $\begin{array}{l}\text { Cruz, C. C., \& Santos, K.P. } \\
2021 .\end{array}$ & $\begin{array}{l}\text { A humanização do parto no } \\
\text { Hospital Maternidade Mãe } \\
\text { Luzia, em Macapá -AP }\end{array}$ & Pesquisa qualitativa. & $\begin{array}{l}\text { Traz sobre os direitos da parturiente, } \\
\text { a humanização, os avanços e os } \\
\text { desafios encontrados. }\end{array}$ \\
\hline
\end{tabular}

Fonte: Autores (2021).

A atuação do enfermeiro obstetra é de suma importância na assistência ao parto normal, visto que, desde o ano de 1998, o Ministério da Saúde vem qualificando o profissional para prática no atendimento e acompanhamento da gestação de baixo e 
alto risco. Com o objetivo de humanizar o serviço de saúde e reduzir medidas desnecessárias, como, a realização de cesarianas sem indicação, a assistência do enfermeiro ao parto normal humanizado está ligada diretamente à valorização da parturiente, respeitando seu corpo e suas características, usando apenas medidas que sejam necessárias, certificando seus direitos em todos os seus aspectos (Martins, 2018).

O nascimento de forma humanizada traz, além de mudanças no processo de trabalho dos profissionais da saúde, no respeito à individualidade de cada parturiente e às suas escolhas durante todo o processo. O profissional deve estar apto a perceber o seu campo de atuação para além das técnicas de intervenções e das tecnologias do cuidado em saúde, o que resulta em um grande desafio para mudar o cenário da assistência ao parto (Silva, 2020).

O enfermeiro é fundamental na assistência da parturiente, considerando que a recebe na hora que é necessário o seu atendimento, escuta seus anseios quanto ao medo do parto, proporciona amparo e conforto no decorrer do processo do parto, estimulando-a a reconhecer seu papel de protagonista, sendo capaz de utilizar técnicas transformadoras do meio em que atua, tornando a ciência que aprendeu em exemplos humanistas e levando em conta os direitos da mulher à maternidade segura $\mathrm{e}$ prazerosa. São várias as atuações e intervenções realizadas pelo enfermeiro, que contempla a mulher nos direitos sexuais e reprodutivos, pré concepção, gestação, pré parto, parto e puerpério, cuidados e manejo do recém-nascido e do abortamento, além do processo de parto, sendo um tema bem discutido sob sua percepção profissional. Contudo, tem-se notado um número não tão expressivo na literatura, de estudos que objetivam conhecer a satisfação das puérperas frente a mesma assistência de enfermagem oferecida, haja vista que uma assistência considerada humanizada pela enfermagem pode ser vivenciada pelas parturientes de uma forma diferente (Barros, 2018).

Essas intervenções e condutas têm o potencial de interferir no cuidado fornecido à mulher durante o parto, desprezando os seus direitos e de sua família nesse processo. Diante disso, a Organização Mundial de Saúde (OMS), bem como o Ministério da Saúde e alguns órgãos não governamentais, propôs mudanças. Essas enfatizam o cuidado oferecido às mulheres, incluindo o resgate do parto natural. Isso também têm impulsionado a atuação de enfermeiras obstetras e equipes qualificadas na assistência à gestação e ao parto, além de ações de incentivo para que o parto seja tratado como um processo fisiológico, conduzido a partir da perspectiva da humanização (Possati, 2017).

A participação ativa do profissional enfermeiro/médico obstetra na assistência do trabalho de parto e nascimento habilita o trabalho em equipe e colabora para um cuidado humanizado. As enfermeiras obstétricas baseiam sua atuação nas boas práticas recomendadas pelo Ministério da Saúde e Organização Mundial da Saúde (OMS), realizando um cuidado oferecem diversos benefícios maternos e neonatais. A importância no cuidado proporciona conforto e ajudam no processo de parto e nascimento, ao mesmo tempo em que estimulam a autonomia e o protagonismo da mulher durante todo o processo (Lima, 2020)

Os principais desafios enfrentados para a prática da humanização no parto e nascimento é a dificuldade de alguns profissionais de saúde em se enquadrar-se dentro das práticas humanizadas. Outro obstáculo enfrentado é baseado na sua formação profissional, que é guiada no modelo biomédico, que muitas vezes está centrado na doença e restringe-se aos fatores biológicos, ou seja, um atendimento que utiliza métodos tecnológicos, não priorizando as práticas humanizadas, causando consequentemente, um despreparo da equipe nos atendimentos (Ahlert \& Lohmann, 2019).

Enfermeiras obstetras possuem especialização, e determinada qualificação o processo fisiológico do nascimento, colaborando para a sua evolução natural, reconhecendo e corrigindo os desvios da normalidade, e encaminhando aquelas que necessitem de uma assistência especializada (Santana, 2019).

A enfermeira obstetra precisa seguir um modelo de assistência que resgate valores como o protagonismo, a individualidade, a privacidade e a autonomia da mulher, visando a promoção de partos saudáveis, eliminando intervenções desnecessárias e oferecendo outras comprovadamente benéficas. Dentre os cuidados, essa profissional deve estar alerta às queixas e outros sinais que possam indicar qualquer intercorrência (Alves, 2018). 
A inserção dos enfermeiros obstetras na equipe multiprofissional no cenário de humanização do processo de parturição é essencial, pois a formação desse profissional é pautada no cuidado e na valorização dos aspectos fisiológicos do parto, valoriza e considera os direitos da mulher, promovendo o vínculo entre profissional e paciente, podendo assim, evitar as intervenções desnecessárias e contribuir para a redução dos índices de mortalidade materna (Lima, 2017).

A atuação do enfermeiro no parto natural na perspectiva da humanização, tem como finalidade identificar as ações cuidadoras que o enfermeiro implementa no parto normal, verificar os fatores que interferem na humanização da assistência de enfermagem no parto natural e ampliar a visão dos enfermeiros em relação às reações percebidas pelas gestantes após o recebimento da assistência humanizada (Pereira, 2016).

O papel da Equipe de Enfermagem é fundamental durante todo o processo, o qual consiste em assistir a puérpera nesse período, além de emergir conhecimento sobre as técnicas do parto, disposta a ajudar e identificar qualquer alteração anormal caso haja. A mesma deve saber respeitar as necessidades e cultura já vividas pela paciente. A humanização durante o parto traz práticas que irão proporcionar a parturiente uma melhor garantia de segurança, confiança, certezas e fortalecimento de laços, pois é um instante que trata não só de mudanças físicas, mas psicológicas, que envolvem o emocional, correlacionando a interferência do pai nesse momento, reforçando a importância dos laços familiares (Santos, 2020).

O enfermeiro obstetra não pode garantir o resultado final do processo de parturição, mas pode salvaguarda-se de zelo e cuidados, evitando expor a mulher e o bebê a riscos desnecessários ao exercer suas atividades na assistência ao parto. As condutas do profissional de Enfermagem podem marcar a vida dos pacientes, causando satisfação ou descontentamento. Estes profissionais devem estabelecer um diálogo que esclareça a finalidade da assistência, oportunize a liberdade de opção e descubra as dúvidas e expectativas dos pacientes (Mota, Waltrick \& Barbosa, 2019).

Embora existam esses esforços a fim de aperfeiçoar toda a assistência à mulher em processo de parturição, observa-se que é necessária sensibilização dos profissionais de saúde para o exercício da atenção, do diálogo, do acolhimento e da comunicação com a parturiente, para atingir o ideal de humanização. A participação ativa dos profissionais de enfermagem nesse processo é importante, pois como integrantes da equipe de saúde devem se utilizar, além dos conhecimentos técnico-científicos, do respeito à mulher como ser único, com desejos e direitos, no intuito de promover a assistência humanizada. (Piler, 2020).

\section{Conclusão}

Muitas mulheres ainda carregam consigo a ideia de que a dor do parto natural é necessária para prepará-las enquanto mães, pois nesse momento é vital a demonstração de força, maturidade, capacitação, porém esse conceito tornou-se ultrapassado, já que a mães não precisam viver a dor em sua forma mais intensa para estar preparada para cumprir o seu papel. Por isso, as mulheres passam a optar pelo parto cesáreo, ao qual é caracterizado por ser uma intervenção cirúrgica que vem crescendo e tomando espaço ao longo dos anos, o mesmo se dá com aplicação de anestesia e com isso a mãe não passa a dor do parto. Porém, ela precisa de mais tempo no hospital, pois a dor ocorre com o passar do efeito anestésico, onde a mesma fica restrita a locomoção, movimentação e o principal que é cuidar inteiramente do seu filho, além dos riscos inerentes ao procedimento cirúrgico.

Para reduzir a ocorrência de partos cesáreos, utiliza-se uma ferramenta chamada humanização do parto. Esse, se dá por meio de práticas voltadas a parturiente, onde lhe é oferecido cuidado, carinho, atenção e com isso é possível reduzir essa dor. As medidas aplicadas para essa humanização é o direito que se dá a parturiente de escolher o seu acompanhante, a possibilidade da escolha da via do parto, o local, a posição em que ocorrerá, a realização de massagens, musicoterapia e etc., um leque de opções que levam ao relaxamento, aumento da autossatisfação e a confiança da mulher.

O desenvolvimento do trabalho permitiu compreender que o enfermeiro obstetra possui conhecimentos técnicos necessários para o desenvolvimento dessa prática, principalmente por ser situação em que o médico não se encontra totalmente presente ou por não poder estar durante todo o processo em função do tempo que se leva. 
Com isso, o profissional poderá utilizar da sua preparação profissional para atuar diretamente com a gestante, prestando todos os cuidados e esclarecimentos necessários, conversando, tranquilizando, indicando as opções e estimulando a parturiente para que ela enfrente o parto com conforto, segurança e menos dor.

Este estudo poderá servir como protótipo para a realização de trabalhos futuros para um melhor entendimento das representações e práticas dos profissionais enfermeiros quanto ao parto humanizado, visando não só um melhor resultado na assistência como também, maior satisfação e benefícios para essas mulheres.

\section{Referências}

Ahlert, E. C., Alves, A. M., \& Lohmann, P. M. (2019). A percepção dos profissionais da área obstétrica de dois hospitais do vale do taquari sobre o parto humanizado. Revista Destaques Acadêmicos. Lajeado. 11(3).

Alves, T. T. M. (2018). Atuação da enfermeira obstetra no desenrolar do trabalho de parto e parto. Revista Enfermagem Health Care [online]. 7(1), 41-50.

Barbosa, E. M. G. (2018). Necessidades de autocuidado no período pós-parto identificadas e grupos de puérperas e acompanhantes. Revista de enfermagem e atenção à saúde. 7(1), 164-176.

Barros, F. R. B. (2018). Percepção das puérperas manauaras frente à assistência de enfermagem no preparo do trabalho de parto e nascimento. Enfermagem Foco. 9(1), 76-8.

Braz, I. M. A. (2019). Interdisciplinaridade na assistência ao parto: percepção dos enfermeiros obstetras. Revista enfermagem UFPE online. 13.

Campos, B. C., \& Rodrigues, O. M. P. R. (2015). Depressão Pós-Parto Materna: Crenças, Práticas de Cuidado e Estimulação de Bebês no Primeiro Ano de Vida. Revista Psico. Porto Alegre. 46(4), 483-492.

Cananéa, B. A., Coutinho, L. C., \& Meirelles, L. X. (2020). A parturição na perspectiva das tecnologias não invasivas de cuidado de enfermagem: narrativas de vida. Brazilian Journal of Development. 6(11), 87145-87156.

Cruz, C. C., \& Santos, K. P. (2021). A humanização do parto no Hospital Maternidade Mãe Luzia, em Macapá - AP. Brazilian Journal of Development. Curitiba. $7(2), 14557-14571$.

Lima, M. M. (2020). Enfermeiras obstétricas no processo de parturição: percepção das mulheres. Revista enfermagem UERJ.

Lima, P. C. (2017). A vivência de adolescentes assistidas por enfermeiros obstetras durante o processo de parturição. Revista de Enfermagem do Centro-Oeste Mineiro. 7.

Martins, D. O. (2018). Percepção das puerperas sobre o parto normal humanizado. Temas em Saúde. João Pessoa. 844-860.

Mendonça, S. S. (2021). Entre riscos fisiológicos e emocionais: a humanização do parto em uma maternidade pública carioca. Teoria e Cultura. 16(1), 153-166.

Mota, B. R., Waltrick, M. A. M., \& Barbosa, T. M. (2019). Mulheres em puerpério: representação social sobre o atendimento da enfermagem no momento do parto. Saúde. \& Transformação. Social. Florianópolis. 10(1/2/3). 89-102

Nascimento, E. R. (2020). Desafios da assistência de enfermagem ao parto humanizado. Caderno de Graduação-Ciências Biológicas e da Saúde-UNIT-Sergipe. $6(1), 141$.

Pereira, S. S. (2016). Parto natural: a atuação do enfermeiro diante da assistência humanizada. Tempus, ACTAS de saúde coletiva. 10(3), 199-213.

Piler, A. A. (2020). Cuidados no processo de parturição sob a ótica dos profissionais de enfermagem. Texto \& Contexto Enfermagem. 29.

Possati, A. B. (2017). Humanização do parto: significados e percepções de enfermeiras. Escola Anna Nery Revista de Enfermagem. 21 (4), 1-6.

Santana, A. T. (2019). Atuação de enfermeiras residentes em obstetrícia na assistência ao parto. Rev. Bras. Saúde Mater. Infant. Recife. 19(1), 145-155.

Santos, J. C. M. (2020). A percepção das puérperas sobre o parto vaginal humanizado assistido pela equipe de Enfermagem. Research, Society and Development. $9(10), 2020$.

Silva, N. G. T. (2021). As demandas emocionais na gestação e os seus desdobramentos no processo de parto. Research, Society and Development. 10(9), e36810917884-e36810917884.

Silva, M. J. (2020). O movimento pela humanização do parto e nascimento no Brasil: o impacto em Uberlândia segundo a percepção dos enfermeiros. Braz. J. Hea. Rev. Curitiba. 3(4), 7614-7634. 